\title{
ENRIQUECIMIENTO AMBIENTAL Y SU EFECTO EN LA EXHIBICIÓN DE COMPORTAMIENTOS ESTEREOTIPADOS EN JAGUARES (PANTHERA ONCA) DEL PARQUE ZOOLÓGICO "YAGUAR XOO”, OAXACA
}

\author{
Citlalli CASTILlO-GUEVARA, ${ }^{1 *}$ Karime UNDA-HARP, ${ }^{2}$ CaRlos LARA $^{1}$ \\ \& JUAN CARLOS SERIO-SILVA ${ }^{3}$ \\ ${ }^{1}$ Laboratorio de Biodiversidad, Centro de Investigación en Ciencias Biológicas. Universidad \\ Autónoma de Tlaxcala. Km 10.5 Autopista Tlaxcala-San Martín Texmelucan San Felipe Ixtacuixtla, \\ Tlaxcala.90120. México. <guevaracc@yahoo.com.mx>; <laracar2000@hotmail.com> \\ ${ }^{2}$ Maestría en Conservación y Manejo de Vida Silvestre, Instituto Internacional en Conservación \\ y Manejo de Vida Silvestre, Universidad Nacional. Apartado 1350-3000, Heredia, Costa Rica. \\ <karimeunha@gmail.com> \\ ${ }^{3}$ Red de Biología y conservación de vertebrados. Instituto de Ecología, A.C. Km. 2.5 Carretera antigua \\ a Coatepec 351, Congregación El Haya, Xalapa, Veracruz 91070, México. \\ <juan.serio@inecol.edu.mx> \\ * Autor corresponsal: <guevaracc@yahoo.com.mx>
}

Castillo-Guevara, C., K. Unda-Harp, C. Lara \& J. C. Serio-Silva. 2012. Enriquecimiento ambiental y su efecto en la exhibición de comportamientos estereotipados en jaguares (Panthera onca) del Parque Zoológico “Yaguar Xoo”, Oaxaca. Acta Zoológica Mexicana (n. s.), 28(2): 365-377.

RESUMEN. El desarrollo de programas de enriquecimiento ambiental para animales en cautiverio promueve el bienestar animal aumentando la actividad física, reduciendo el estrés y, previniendo o reduciendo trastornos como las estereotipias o comportamientos anormales. En el Parque Zoológico Yaguar Xoo se diseñó y aplicó un programa a cuatro jaguares (Panthera onca). Fueron dos machos (de cuatro y diez años de edad) y dos hembras (de cuatro y diez años, respectivamente). Se aplicaron estímulos ocupativos (mordedera y garrafón con piedras), alimenticios (pollo, carne de res, pescado, alimento comercial “Whiskas ${ }^{\circledR}$ ”) y sensoriales (grabaciones de vocalizaciones de jaguar Panthera onca, puma Puma concolor y mono aullador Alouatta palliata, así como sacos con diferentes esencias de menta, naranja, anís, orina de lince Lynx rufus y de ocelote Felis pardis). Con el objetivo de evaluar la eficiencia del programa, mediante la reducción o eliminación de las estereotipias (balanceo, paseo y vocalización), se midieron los patrones de comportamiento presentados en los sujetos de estudio durante tres diferentes etapas: (1) previa al enriquecimiento (26 días), (2) aplicación del enriquecimiento ambiental (34 días) y, (3) posterior al enriquecimiento (22 días). Como resultado de la aplicación del programa encontramos una disminución significativa en la ejecución de estereotipias y un incremento en los comportamientos normales en los individuos de estudio. Para los cuatro jaguares el número de comportamiento normales exhibidos (independientemente de que fueran individuales o sociales) fue significativamente distinto

Recibido: 24/10/2011; aceptado: 11/04/2012. 
entre las tres etapas del estudio $\left(\mathrm{F}_{2,306}=4.215, p=0.0156\right)$. En la etapa posterior al enriquecimiento la frecuencia de comportamientos normales fue similar a la etapa previa al enriquecimiento ( $p=0.4680$ ). El balanceo (9.57\%) disminuyó drásticamente una vez que se aplicó el enriquecimiento ambiental $(0.96 \%)$ y, esta tendencia se mantuvo aun cuando el enriquecimiento fue retirado (3.88\%) $\left(X^{2}=102.9\right.$, $p<0.001)$. El paseo (1.96\%) también disminuyó drásticamente una vez que se aplicó el enriquecimiento ambiental $(0.36 \%)$ y, una vez que el enriquecimiento fue retirado, el porcentaje se incrementó a niveles similares con la etapa previa $(1.26 \%)\left(X^{2}=12.6, p<0.001\right)$. La vocalización (3.06\%) disminuyó drásticamente una vez que se aplicó el enriquecimiento ambiental $(0.48 \%)$, sin embargo fue similar en las etapas previa y posterior al enriquecimiento $(3.49 \%)\left(X^{2}=21.14, p<0.001\right)$. Esto demuestra que la aplicación del programa tuvo un efecto positivo al tiempo de evaluación en el comportamiento de los felinos, al disminuir la frecuencia de estereotipias.

Palabras clave: Cautiverio, enriquecimiento ambiental, felinos, estereotipia, bienestar animal.

Castillo-Guevara, C., K. Unda-Harp, C. Lara \& J. C. Serio-Silva. 2012. Environmental enrichment and its effects on exhibit stereotypic behaviors in jaguars (Panthera onca) from the "Yaguar Xoo" Zoological Park, Oaxaca. Acta Zoológica Mexicana (n. s.), 28(2): 365-377.

ABSTRACT. The development of environmental enrichment programs to captive animals, promotes the animal welfare by increasing their physical activity as well as diminishing the stress, and preventing or reducing abnormal or stereotypic behaviors. In the Yaguar Xoo Zoological Park we designed and applied an environmental enrichment program to four captive jaguars (Panthera onca). Were two males (four and ten years old) and two females (four and ten years respectively). Enrichment items (teething and bag with stones) were applied, food (chicken, beef, fish, food shopping "Whiskas ${ }^{\circledR}$ ”) and sensory (recordings of vocalizations of jaguar Panthera onca, puma Puma concolor and howler monkey Alouatta palliata, and as bags with different essences of mint, orange, anise, lynx Lynx rufus and ocelot Felis pardis urine). Our goal was to evaluate the program's efficiency on the reduction or elimination of stereotypic behaviors (swinging, pacing, and vocalization). The exhibit behaviors were evaluated through three different phases: (1) the pre-enrichment phase (26 days), (2) the environmental enrichment application phase (34 days), and (3) the post-enrichment phase (22 days). As a result of the application program found a significant decrease in the performance of stereotypies and an increase in behavior normal individuals studied. For these four jaguars, the number of displayed normal behavior (whether they were individual or social) was significantly different among the three stage of the study $\left(F_{2,306}=4.215\right.$, $p=0.0156)$. In the post-enrichment frequency of normal behavior was similar to the pre-enrichment $(p=0.4680)$. The swinging $(9.57 \%)$ decreased dramatically once applied environmental enrichement $(0.96 \%)$ and, this trend continued even though the enrichment was removed (3.88\%) $\left(X^{2}=102.9, p\right.$ $<0.001)$. The pacing (1.96\%) also fell dramatically once applied environmental enrichment (0.36\%) and, once the enrichment was removed, the percentage increased to levels similar to the previous stage (1.26\%) $\left(X^{2}=12.6, p<0.001\right)$. The vocalization (3.06\%) decreased dramatically once applied environmental enrichment (0.48\%), however was similar in the stages before and after the enrichment (3.49\%) $\left(X^{2}=21.14, p<0.001\right)$. In conclusion, the application of the environmental enrichment enhance captive jaguars' well-being by stimulating active behaviors and reducing stereotypical behaviors.

Key words: Captivity, environmental enrichment, felids, stereotypies, animal welfare.

\section{INTRODUCCIÓN}

El mantenimiento del comportamiento natural es un ideal para los animales en cautiverio que están en programas de conservación, investigación o educación, enfoca- 
dos principalmente al aumento del conocimiento sobre estas especies (WAZA 2005). De esta manera, para que la cautividad sea considerada como efectiva en brindar bienestar para los animales confinados, éstos deberían exhibir los comportamientos usualmente presentados en sus ambientes naturales (Mason et al. 2007). Sin embargo, cuando estos animales no cuentan con los estímulos necesarios para regular sus sistemas biológicos, se presentan anormalidades en las respuestas fisiológicas y conductuales, lo que provoca problemas de salud y fallas reproductivas.

Los zoológicos utilizan técnicas de enriquecimiento ambiental para promover el bienestar animal en cautiverio, identificando y brindando el estímulo ambiental necesario para un óptimo desarrollo psicológico y fisiológico (Shepherdson 1998, Mellen \& McPhee 2001) y de esta manera reducir o eliminar estereotipias en los individuos en cautiverio. El enriquecimiento reduce substancialmente los comportamientos estereotipados exhibidos por mamíferos en zoológicos (Shyne 2006). Una estereotipia es cualquier patrón de movimiento que (1) es realizado en repetidas ocasiones, (2) es relativamente invariable en forma, y (3) no tiene una función u objetivo aparente (Mason 1991). Shyne (2006) demostró que la frecuencia en que los animales presentan estereotipias es una medida sensible sobre la efectividad de una condición de enriquecimiento. Las estereotipias han demostrado ser influenciadas por muchos factores en cautiverio como el tamaño y complejidad del encierro (Mallapur \& Chellam 2002). Las estereotipias han sido ampliamente estudiadas en carnívoros, y en particular, el paseo esterotipado, reingestión, regurgitación, coprofagia y aseo excesivo son observadas en felinos (Mallapur 1999).

Algunos estudios realizados en zoológicos de Estados Unidos han evaluado los efectos del enriquecimiento ambiental en felinos. Por ejemplo, un estudio realizado en el Zoológico de Atlanta, demostró que el enriquecimiento alimenticio (cazar presas vivas) en leones (Panthera leo) y tigres (Panthera tigris), elevó la frecuencia de comportamientos normales de alimentación y redujo las estereotipias (Bashaw et al. 2003). En el Zoológico de Montgomery, Alabama, se realizó una comparación de varios tipos de enriquecimiento para seis especies de felinos (guepardo, Acinonyx jabatus; puma, Puma concolor; jaguar, Panthera onca; león, Panthera leo; ocelote, Felis pardalis; tigre, Panthera tigris). Los huesos, las especias y el pescado congelado fueron los mejores estímulos para incrementar su actividad, además de ser económicos y fáciles de emplear (Skibiel et al. 2007).

En México, hay pocos ejemplos de la implementación de programas de enriquecimiento ambiental para animales en cautiverio. Por ejemplo, el zoológico Africam Safari, Puebla, implementa desde el 2006 un programa a un elefante hembra de edad avanzada, para estimularla físicamente y eliminar las estereotipias (como el "bamboleo”, comportamiento común en elefantes en cautiverio), y de esta manera lograr que su comportamiento sea más atractivo para el público (Martínez 2006). Dentro del Zoo Parque Loro, Puebla, también se implementó un programa en primates, osos ne- 
gros y felinos, estimulando uno o diferentes sentidos a la vez (K. Unda, Com. Pers.). De acuerdo con el Programa General de Desarrollo 2007-2012, los Zoológicos de Chapultepec, San Juan de Aragón y Los Coyotes realizan actualmente programas de enriquecimiento ambiental que tienen la finalidad de estimular comportamientos normales y mantener su bienestar; y durante el 2007 se desarrollaron un total de 121433 actividades de enriquecimiento en 2179 individuos de 116 especies diferentes (DGZCM 2006, 2007).

Actualmente, existen pocos estudios sobre programas de enriquecimiento aplicados a felinos en cautiverio en el país. Dos trabajos se han llevado a cabo específicamente en Panthera onca, el primero de ellos se realizó en el Parque Museo de la Venta, Tabasco, donde utilizando tres tipos de estímulos (sensoriales, alimenticios y ocupativos), Granda-Serrano \& Romo-Graniel (2004) registraron un aumento en el comportamiento social normal de los jaguares así como una disminución de las estereotipias. El segundo estudio se realizó en el Zoológico Regional Miguel Álvarez del Toro, Chiapas, donde se obtuvo un aumento significativo en la actividad y comportamientos normales, y reducción de peso en los jaguares (Aguilar \& León 2005).

El Parque Zoológico Yaguar Xoo, Oaxaca, tiene en exhibición 70 animales y recibe aproximadamente 4680 visitantes anualmente. En el área de felinos hay dos recintos designados a dos parejas de jaguar (Panthera onca) que presentan estereotipias (balanceo, paseo y vocalización), sin embargo, este zoológico no cuenta con algún programa de enriquecimiento ambiental en ninguna de sus áreas. Por ello se planteó implementar un programa de enriquecimiento para aminorar estos comportamientos anormales, y promover la salud física y conductual de los jaguares. El objetivo del presente trabajo fue la aplicación y evaluación de la eficiencia de un programa de enriquecimiento ambiental en los cuatro jaguares del Parque Zoológico Yaguar Xoo. Para ello, (1) se identificó la presencia de estereotipias, (2) se aplicó un programa de enriquecimiento ambiental y, (3) se evaluó si el comportamiento de los cuatro jaguares fue modificado por la aplicación del programa de enriquecimiento ambiental, aumentando los comportamientos naturales y reduciendo las estereotipias. Mediante la implementación del programa se esperaba que los comportamientos anormales o estereotipados observados en los cuatro ejemplares de jaguar en cautiverio, lograran reducirse ó eliminarse en corto plazo.

\section{MATERIALES Y MÉTODOS}

Sujetos de estudio. El Parque Zoológico Yaguar Xoo se localiza en la población de San Francisco Tanivet, Distrito de Tlacolula, a 42 kilómetros de la ciudad de Oaxaca. Los jaguares se exhiben al público visitante durante cuatro horas (9000-1300 h) de lunes a viernes y, durante ocho horas (9000-1700 h) sábado y domingo (ver Cuadro 1 para las características individuales). Cada pareja se encuentra alojada en un recinto exhibidor de $923 \mathrm{~m}^{2}$ y $146 \mathrm{~m}^{2}$, respectivamente. Las observaciones para el estudio se 
Cuadro 1. Características individuales de los jaguares (Panthera onca) del Parque Zoológico Yaguar Xoo.

\begin{tabular}{|c|c|c|c|c|c|c|}
\hline Recinto & Nombre & Sexo & $\begin{array}{l}\text { Edad aproximada } \\
\text { (años) }\end{array}$ & $\begin{array}{l}\text { Años en la } \\
\text { institución }\end{array}$ & Origen & Coloración \\
\hline 1 & Gitano (A) & Macho & 4 & 4 & Nacimiento & Amarillo \\
\hline 1 & Zulema (B) & Hembra & 4 & 4 & Nacimiento & Amarillo \\
\hline 2 & Mocho (C) & Macho & 10 & 6 & $\begin{array}{c}\text { Proyecto } \\
\text { Balam } \\
\text { Puebla }\end{array}$ & Melánico \\
\hline 2 & Gitanilla (D) & Hembra & 10 & 3 & $\begin{array}{c}\text { Proyecto } \\
\text { Balam } \\
\text { Puebla }\end{array}$ & Amarillo \\
\hline
\end{tabular}

realizaron en ambos recintos exhibidores, dos días al azar entre semana y los fines de semana.

Observaciones. Se realizaron observaciones ad libitum durante dos semanas (1024 de mayo, 2008), registrando los comportamientos y aprendiendo a identificar a los individuos. Posteriormente, las observaciones conductuales se realizaron en tres etapas. (1) Etapa previa al enriquecimiento ambiental (3 de Junio al 3 de Agosto, 2008): donde utilizando la técnica de muestreo animal focal instantáneo (Martin \& Bateson, 1997), se registró el repertorio conductual de los jaguares y con base en esto los comportamientos fueron clasificados en individuales, sociales y estereotipados (Cuadro 2) de acuerdo a lo propuesto por Granda-Serrano \& Romo-Graniel (2004), McPhee (2002) y Skibiel et al. (2007). En cada sesión de observación se registró para cada jaguar el número de comportamientos exhibidos de cada tipo, y al final de la etapa con los datos obtenidos se estimó el porcentaje total para cada conducta. El registro fue de cuatro horas por día, una hora para cada uno de los individuos, los comportamientos se registraron en intervalos de tiempo de cinco minutos. Esta etapa tuvo una duración de 26 días. (2) Etapa durante el enriquecimiento ambiental (12 de Agosto al 19 de Octubre, 2008): se aplicó el programa de enriquecimiento de acuerdo al tipo de estereotipias identificadas en la etapa previa, y éste consistió de estímulos ocupativos (mordedera y garrafón con piedras), alimenticios (pollo, carne de res, pescado, alimento comercial "Whiskas ${ }^{\circledR}$ ”) y sensoriales (grabaciones de vocalizaciones de jaguar Panthera onca, puma Puma concolor y mono aullador Alouatta palliata, así como sacos con diferentes esencias de menta, naranja, anís, orina de lince Lynx rufus y de ocelote Felis pardis). Estos estímulos fueron previamente utilizados en felinos por McPhee (2002), Granda-Serrano \& Romo-Graniel (2004), Skibiel et al. (2007). Los estímulos usados para el enriquecimiento fueron ofrecidos sólo durante nuestro periodo de registro. Utilizando el mismo método de observación se registraron los comportamientos tomando como referencia los mismos patrones de comportamiento 
Cuadro 2. Patrones de comportamiento normal (individual y social), y comportamientos anormales o estereotipias de acuerdo a McPhee (2002)*; Granda-Serrano \& Romo-Graniel (2004)** y Skibiel et al. $(2007)^{* * *}$.

\begin{tabular}{|c|c|}
\hline Comportamientos individuales & Definición \\
\hline Alerta* & $\begin{array}{l}\text { Estado de alerta, atento; puede estar parado o } \\
\text { recostado }\end{array}$ \\
\hline Estiramiento* & $\begin{array}{l}\text { Las extremidades traseras estiradas, las } \\
\text { extremidades delanteras estiradas abajo del } \\
\text { cuerpo. Extremidades delanteras estiradas } \\
\text { delante del cuerpo, extremidades traseras } \\
\text { estiradas abajo del cuerpo }\end{array}$ \\
\hline Alimentación** & El individuo está ingiriendo alimento \\
\hline Descanso** & $\begin{array}{l}\text { No locomoción ni conducta social, reposo con } \\
\text { ojos abiertos o cerrados }\end{array}$ \\
\hline Flehmen** & $\begin{array}{l}\text { El macho (y con mejor frecuencia la hembra) } \\
\text { huele la orina durante algunos segundos, alza } \\
\text { la cabeza y abre la boca levantando los carrillos } \\
\text { y retrayendo las orejas; a la vez, balancea la } \\
\text { cabeza horizontalmente, permaneciendo algunos } \\
\text { segundos en esta posición }\end{array}$ \\
\hline Giros** & $\begin{array}{l}\text { Movimientos dorso-ventrales, de flanco derecho } \\
\text { a izquierdo, llevando las extremidades anteriores } \\
\text { al rostro }\end{array}$ \\
\hline Limpieza** & $\begin{array}{l}\text { Con la lengua repasan su cuerpo en repetidas } \\
\text { ocasiones }\end{array}$ \\
\hline Locomoción** & $\begin{array}{l}\text { Movimientos repentinos, correr, saltar, agitar o } \\
\text { menear la cabeza y mantenerse en pie después } \\
\text { del descanso }\end{array}$ \\
\hline Marcaje por orina** & $\begin{array}{l}\text { Con frecuencia orinan sobre las paredes, suelo o } \\
\text { troncos }\end{array}$ \\
\hline Nado*** & $\begin{array}{l}\text { Cualquier actividad realizada cuando el animal } \\
\text { está en el agua }\end{array}$ \\
\hline Bostezo & $\begin{array}{l}\text { Acción incontrolada de abrir la boca, con } \\
\text { separación muy amplia de las mandíbulas, para } \\
\text { realizar una inspiración profunda a la que sigue } \\
\text { una expiración de algo menos de lo inhalado, } \\
\text { con cierre final }\end{array}$ \\
\hline Defecar & Proceso biológico de eliminación de las heces \\
\hline Olfateo & Oler a otros individuos u objetos \\
\hline Interacción con objeto & $\begin{array}{l}\text { Acción ejercida entre el jaguar y cierto objeto } \\
\text { en particular, ya sea mordiéndolo, marcándolo, } \\
\text { olfateándolo o jugueteando con él }\end{array}$ \\
\hline
\end{tabular}


Cuadro 2. Continuación.

\begin{tabular}{|c|c|}
\hline Marcaje por uñas & $\begin{array}{l}\text { Afilar las uñas vigorosamente en un área u } \\
\text { objeto, dejando así su olor para indicar la } \\
\text { posesión de su territorio }\end{array}$ \\
\hline Purga & Acción de ingerir forraje y regurgitar \\
\hline Comportamientos sociales & Definición \\
\hline Acercamiento** & $\begin{array}{l}\text { Un individuo frota con su cabeza levemente a } \\
\text { otro individuo }\end{array}$ \\
\hline Acicalamiento** & $\begin{array}{l}\text { Uno de los individuos lame la zona del cuello, } \\
\text { nuca, orejas y flancos de otro individuo }\end{array}$ \\
\hline Agresión** & $\begin{array}{l}\text { El individuo agresor gruñe fuertemente, baja las } \\
\text { orejas y levanta excesivamente los carrillos }\end{array}$ \\
\hline Cópula** & $\begin{array}{l}\text { Después de los movimientos pélvicos el macho } \\
\text { pega totalmente sus genitales a los de la hembra } \\
\text { y se queda unido por un instante a ésta, en ese } \\
\text { momento el macho ruge y muerde fuertemente la } \\
\text { nuca de la hembra, inmediatamente después da } \\
\text { un salto para separarse de ella y permanece muy } \\
\text { cerca de ésta, la hembra por su parte, reacciona } \\
\text { dando un giro en dirección del macho, sin } \\
\text { agredirlo }\end{array}$ \\
\hline Jugueteo** & $\begin{array}{l}\text { Se inicia cuando cada uno de los individuos } \\
\text { acecha a otro, intercambiando manotazos y } \\
\text { mordidas superficiales no agresivas }\end{array}$ \\
\hline Monta** & $\begin{array}{l}\text { La hembra adopta la posición de lordosis, el } \\
\text { macho baja sus cuartos traseros y lleva a cabo } \\
\text { una serie de movimientos pélvicos, pero la } \\
\text { cópula no se consuma, no hay eyaculación }\end{array}$ \\
\hline Persecución** & $\begin{array}{l}\text { La hembra y el macho caminan juntos, siendo } \\
\text { ella la que toma la iniciativa a una distancia no } \\
\text { menor de } 5 \mathrm{~m}\end{array}$ \\
\hline Rechazo a monta** & $\begin{array}{l}\text { El macho intenta montar a la hembra y ésta da } \\
\text { un giro brusco y gruñe, en señal de rechazo; o la } \\
\text { hembra no adopta la posición de lordosis }\end{array}$ \\
\hline Solicitud de permiso** & $\begin{array}{l}\text { El macho se acerca a la hembra y huele los } \\
\text { genitales de ésta y nunca los lame }\end{array}$ \\
\hline Sumisión & $\begin{array}{l}\text { Respuesta de una agresión mostrando } \\
\text { subordinación, aplanan las orejas hacia el } \\
\text { costado }\end{array}$ \\
\hline
\end{tabular}


Castillo-Guevara et al. Enriquecimiento ambiental y su efecto en comportamiento de jaguares

Cuadro 2. Continuación.

\begin{tabular}{ll}
\hline \multicolumn{1}{c}{ Comportamientos anormales o estereotipias } & \multicolumn{1}{c}{ Definición } \\
Balanceo** $^{*}$ & $\begin{array}{l}\text { Movimientos rítmicos repetidos, que coinciden } \\
\text { con la mirada fija sobre un punto, con 3 a } 7\end{array}$ \\
& pasos oscilantes de un lado a otro \\
& Caminar de un lado a otro repetidamente sobre \\
Paseo** $^{* *}$ & $\begin{array}{l}\text { las rutas establecidas de gran distancia, por lo } \\
\text { general de un extremo a otro }\end{array}$ \\
& Emitir sonidos como jadeos, ronroneos o \\
Vocalización & ronquidos de manera constante \\
\hline
\end{tabular}

individual, social y estereotipado que en la etapa uno. Esta etapa tuvo una duración de 34 días. (3) Etapa posterior al enriquecimiento ambiental (22 de Octubre al 21 de Diciembre, 2008): donde no se aplicó ningún enriquecimiento ambiental, se registraron los repertorios conductuales, tomando como referencia los mismos patrones de comportamiento que en las etapas anteriores. Esta etapa tuvo una duración de 22 días.

Análisis estadístico. Dado que el número de estereotipias representó el 10\% del total de los comportamientos exhibidos por los jaguares durante el estudio, la inclusión de estos valores para fines de comparación podía sesgar efectos no significativos del enriquecimiento con respecto a comportamientos normales. Por ello, dado que el enriquecimiento debería implicar por un lado un incremento en la exhibición de comportamientos normales y por el otro una disminución de las estereotipias, los análisis a continuación descritos los evalúan por separado. La variación en el número de comportamientos normales (individuales y sociales) observados en las tres etapas fue analizada con un ANOVA de dos vías (Zar 1999). En el modelo, la etapa (previa, durante y posterior al enriquecimiento) y el tipo de comportamiento (individual y social) fueron factores fijos y, el número de comportamientos observados la variable dependiente. Los datos utilizados para el ANOVA, debido a la falta de ajuste a la normalidad, se transformaron con raíz cuadrada $\left(V_{x}+0.5\right)$ para cumplir con los requerimientos de los análisis paramétricos (Zar 1999). Se realizaron pruebas post hoc para determinar diferencias entre etapas en el número de comportamientos. Los análisis se realizaron a través del paquete estadístico StatView (Abacus Concepts 1996).

La frecuencia de exhibición de cada una de los comportamientos estereotipados identificados (balanceo, paseo y vocalizaciones) fue analizada individualmente para determinar su variación a través de las tres fases del estudio utilizando pruebas de chicuadrado con corrección de Yates (Zar 1999). Los valores observados y esperados de cada una de los comportamientos se compararon utilizando la prueba de los Residuales Ajustados de Haberman para determinar el sentido de las diferencias encontradas en las pruebas de chi-cuadrada $(p<0.001)$. 


\section{RESULTADOS}

El estudio tuvo una duración total de 82 días (328 h de observación): 104 h en la etapa previa al enriquecimiento ambiental (1), $136 \mathrm{~h}$ durante el enriquecimiento ambiental (2) y $88 \mathrm{~h}$ posteriores al enriquecimiento ambiental (3). Se registraron 3969 comportamientos en total, correspondientes a 26 tipos de comportamientos normales (16 individuales y 10 sociales) y tres tipos anormales o estereotipias. Los resultados presentados consideran a los cuatro jaguares observados.

El comportamiento individual que presentó mayor porcentaje de ejecución durante las tres etapas del estudio fue el descanso (media \pm error estándar $=48.50 \pm 7.57$ ) (Cuadro 3). El comportamiento social que presentó mayor porcentaje de ejecución durante las tres etapas del estudio fue el acicalamiento (media \pm error estándar $=1.98$ \pm 0.28 ) (Cuadro 3).

Para los cuatro jaguares el número de comportamiento normales exhibidos (independientemente de que fueran individuales o sociales) fue significativamente distinto entre las tres etapas del estudio $\left(\mathrm{F}_{2,306}=4.215, p=0.0156\right)$. Las pruebas post hoc realizadas para determinar diferencias entre etapas en el número de comportamientos exhibidos por los jaguares demostraron que la mayor frecuencia de comportamientos normales se registró en la etapa enriquecimiento (etapa previa vs etapa enriquecimiento $p=0.0257$, etapa enriquecimiento vs etapa posterior $p=0.0032$ ), mientras que este número no fue distinto entre la etapa previa y posterior (etapa previa vs etapa posterior $p=0.4680$ ). Por otro lado, el número de comportamientos individuales fue mayor que el de los sociales a lo largo del estudio $\left(F_{1,306}=23.230, p=<0.0001\right)$, sin diferencias significativas en su exhibición entre las etapas $\left(F_{2,306}=0.258, p=0.7729\right)$.

Cuadro 3. Porcentaje de ejecución de comportamientos (individuales y sociales) más frecuentes durante las tres etapas del estudio (previa al enriquecimiento, durante el enriquecimiento y posterior al enriquecimiento).

\begin{tabular}{|c|c|c|c|}
\hline \multirow[t]{2}{*}{ Comportamiento } & \multicolumn{3}{|c|}{ Etapas } \\
\hline & Previa al enriquecimiento & $\begin{array}{l}\text { Durante el } \\
\text { enriquecimiento }\end{array}$ & $\begin{array}{l}\text { Posterior al } \\
\text { enriquecimiento }\end{array}$ \\
\hline \multirow[t]{4}{*}{ Individual } & Descanso (50.47\%) & Descanso (34.51\%) & Descanso (60.54\%) \\
\hline & Locomoción (13.10\%) & $\begin{array}{l}\text { Interacción con objeto } \\
(18.78 \%)\end{array}$ & Alerta (11.56\%) \\
\hline & Limpieza (4.39\%) & Locomoción (15.48\%) & Locomoción (9.23\%) \\
\hline & Alerta (3.13\%) & Alerta $(9.42 \%)$ & Limpieza (2.04\%) \\
\hline \multirow[t]{4}{*}{ Social } & Acicalamiento (1.88\%) & Acicalamiento (2.52\%) & Acicalamiento (1.55\%) \\
\hline & Acercamiento (1.41\%) & Acercamiento (1.68\%) & Acercamiento (0.77\%) \\
\hline & Cópula (0.78\%) & Jugueteo (1.44\%) & Agresión (0.58\%) \\
\hline & Persecución (0.62\%) & Agresión (1.20\%) & Jugueteo (0.19\%) \\
\hline
\end{tabular}


El comportamiento estereotipado que presentó mayor porcentaje de ejecución durante las tres etapas del estudio fue el balanceo (media \pm error estándar $=4.80 \pm 2.52$ ). En la etapa previa al enriquecimiento las comportamientos anormales o estereotipias que se presentaron fueron: balanceo (9.57\%), vocalización (3.06\%) y paseo (1.96\%). Durante la aplicación del enriquecimiento disminuyeron las estereotipias: balanceo (0.96\%), vocalización $(0.48 \%)$ y paseo $(0.36 \%)$. En la etapa posterior al enriquecimiento las estereotipias se redujeron en comparación con la etapa previa al enriquecimiento mostrando los siguientes valores: balanceo (3.88\%) y paseo (1.26\%); la vocalización fue similar en ambas etapas previa y posterior al enriquecimiento (3.49\%).

La frecuencia de exhibición de las conductas estereotipadas registradas durante el estudio fue variable a lo largo de las distintas etapas. Los resultados de las pruebas de chi-cuadrado indican que la estereotipia "balanceo" disminuyó drásticamente una vez que se aplicó el enriquecimiento ambiental y, que esta tendencia se mantuvo aun cuando el enriquecimiento fue retirado (Cuadro 4). Por el contrario, aun cuando la estereotipia "paseo" disminuyó con respecto a la etapa previa mientras se realizaba el enriquecimiento, una vez que éste cesó, el número de estas conductas se incrementó nuevamente a niveles similares con la etapa previa (Cuadro 4). El mismo patrón fue registrado para la estereotipia "vocalización”, con disminución con respecto a la etapa previa, una vez que se aplicó el enriquecimiento, pero con un incremento nuevamente al momento de retirarlo (Cuadro 4).

\section{DISCUSIÓN}

El enriquecimiento ambiental ha sido a menudo utilizado para reducir conductas estereotipadas, mejorar la actividad, e incluso reducir las agresiones en diversas especies de animales en cautiverio (McPhee 2002, Bashaw et al. 2003, Swaisgood \&

Cuadro 4. Frecuencia de las tres conductas estereotipadas registradas en los jaguares y residuales ajustados por etapa ( $\chi^{2}$ altamente significativa en los tres casos: $p<0.001$ ). Los residuales (prueba de Haberman, con valores absolutos mayores a 2 son significativamente diferentes: $5 \%$ de la distribución normal).

\begin{tabular}{lcccccc}
\hline \multicolumn{1}{c}{ Etapa } & Balanceo & Residuales & Paseo & Residuales & Vocalizaciones & Residuales \\
\hline $\begin{array}{l}\text { Previa al } \\
\text { enriquecimiento }\end{array}$ & 122 & $\mathbf{8 . 1 4}$ & 25 & $\mathbf{2 . 7}$ & 39 & $\mathbf{2 . 1 6}$ \\
$\begin{array}{l}\text { Durante el } \\
\text { enriquecimiento }\end{array}$ & 16 & $-\mathbf{2 . 2 5}$ & 6 & $\mathbf{- 2 . 2 6}$ & 8 & $\mathbf{- 3 . 7 4}$ \\
$\begin{array}{l}\text { Posterior al } \\
\text { enriquecimiento }\end{array}$ & 40 & $-\mathbf{5 . 6 3}$ & 13 & -0.43 & 36 & 1.59 \\
$\chi^{2}$ & 102.9 & & 12.6 & & 21.14 & \\
\hline
\end{tabular}


Sheperdson 2005, Skibiel et al. 2007). Debido al tamaño pequeño de la muestra, nosotros no analizamos diferencias individuales en las conductas estereotipadas, sin embargo, al analizar a los cuatros jaguares juntos nuestros resultados reflejan diferencias estadísticamente significativas en la reducción de las esterotipias después de aplicar el enriquecimiento ambiental. Así, los jaguares del zoológico Yaguar Xoo en Oaxaca, tuvieron un incremento significativo en el número de comportamientos normales exhibidos y una disminución en las estereotipias detectadas durante la primera etapa del estudio y, ambos patrones continuaron en general aun después de aplicado el enriquecimiento. De esta manera, nuestro estudio muestra que la aplicación de un programa de enriquecimiento relativamente barato y fácil de administrar como el utilizado, puede tener efectos positivos en la promoción de comportamientos normales y la reducción de estereotipias.

La disminución de comportamientos repetitivos anormales o estereotipias en animales cautivos representa uno de los principales retos no sólo para los zoológicos en México sino alrededor del planeta. Por ejemplo, Mellen et al. (1998) censaron felinos en 8 zoológicos en Estados Unidos, obteniendo datos para 55 individuos que señalan la presencia de comportamientos estereotipados. Tarou et al. (2005) encontraron mediante la aplicación de cuestionarios a 48 zoológicos albergando 440 primates, que el 13.2\% desplegaban estereotipias. Valores similares de prevalencia de estereotipias en distintos taxa albergados por al menos 200 zoológicos alrededor del mundo, son reportados en una revisión realizada por Mason et al. (2007). Dado este contexto, y considerando que uno de los objetivos principales de cualquier zoológico es ofrecer un ambiente adecuado al animal cautivo promoviendo en lo posible sus comportamientos naturales (WAZA 2005), prácticas relacionadas a lograr esto son fundamentales; y es aquí donde el enriquecimiento ambiental juega un papel importante.

En la práctica, las estereotipias en animales pueden algunas veces ser ignoradas o erróneamente explicadas (Mason et al. 2007); lo cual es inadecuado para cualquier institución comprometida con la educación pública o el cuidado animal, tal como sucede con los zoológicos. Por ello, diversas prácticas se han desarrollado para reducirlas o prevenirlas, tales como la selección genética, aplicación de fármacos, el reforzamiento de conductas alternativas, la prevención física o castigo y más comúnmente el enriquecimiento ambiental. Este último ha demostrado ser efectivo y fácil de aplicar, usando estímulos como huesos, especies y pescado congelado en felinos (Skibiel et al. 2007), y está generalmente relacionado con cambios en la estructura y contenido de los encierros. Sin embargo, aspectos relacionados al cuidado, tales como la reducción de ruidos durante el periodo de visitas o los cambios en las interacciones entre el cuidador y el animal pueden ser considerados en esta práctica. Asimismo, el enriquecimiento puede mejorar la experiencia de los visitantes en un zoológico, no sólo porque es más interesante observar animales activos en los encierros (Margulis et al. 2003), sino también porque los visitantes pueden aprender más 
acerca de los animales si éstos despliegan comportamientos parecidos a los exhibidos en la naturaleza (Jones et al. 2005).

Estudios sobre enriquecimiento ambiental en felinos cautivos han sido realizados particularmente en zoológicos de Estados Unidos (por ejemplo Bashaw et al. 2003, McPhee 2005, Skibiel et al. 2007). Sin embargo, en México esta práctica aún está por desarrollarse. En el estudio de Granda-Serrano \& Romo-Graniel (2004), donde se evaluó el efecto del enriquecimiento (ocupativo, alimenticio y sensorial) aun después de su aplicación, encontraron que durante la aplicación de esta práctica en jaguares del zoológico de la Venta en Tabasco, los comportamientos normales aumentaron y las estereotipias disminuyeron. Similarmente, al ser retirado el enriquecimiento, las estereotipias regresaron al valor de la etapa inicial. Nuestros resultados coinciden con lo encontrado por estas autoras, y los datos de ambos estudios sugieren la necesidad de que la aplicación del enriquecimiento debe ser continua. Como lo mencionan previamente otros estudios (Sheperdson 1998, Margulis et al. 2003), nuestros resultados sugieren que el enriquecimiento es una opción "amigable” y barata para disminuir las estereotipias. Bajo esta práctica los animales pueden elegir interactuar o no con los objetos, alimentos y/o estímulos aplicados. Asimismo, tal como ha sido sugerido por Mason et al. (2007), el éxito de un programa de enriquecimiento no sólo debe enfocarse a reducir las estereotipias, sino a reforzar positivamente (incrementar) los comportamientos normales, tal como ocurrió en el presente estudio al reportar un aumento significativo en la exhibición de estos comportamientos por los jaguares.

Los zoológicos representan un valioso recurso para incrementar nuestro entendimiento de cómo los animales responden al cautiverio, pero también importantes centros de prueba para hipótesis relacionadas a determinar si los comportamientos anormales producto de este cautiverio pueden ser eliminados, controlados o estar relacionados con el bienestar del animal. Esfuerzos futuros deben combinar estas aproximaciones.

Agradecimientos. Al director del Parque Zoológico Yaguar Xoo, Oaxaca, por permitir la realización de esta investigación, al MVZ Carlos Gómez por el adiestramiento y, a los trabajadores por el apoyo brindado a lo largo de la aplicación del programa. A Andrea Caiozzi y Dulce Brousset por sus comentarios para el mejoramiento del manuscrito.

\section{LITERATURA CITADA}

Abacus Concepts Inc. 1996. STATVIEW Abacus Concepts, Inc., Berkeley, California, USA.

Aguilar, M. \& A. León. 2005. Enriquecimiento ambiental aplicado a dos ejemplares de jaguar (Panthera onca) en el Zoológico Regional "Miguel Álvarez del Toro”. Tesis de Licenciatura. Universidad de Ciencia y Artes de Chiapas.

Bashaw, M., Bloomsmith, M. \& T. Maple. 2003. To hunt or not to hunt? A feeding enrichment experiment with captive large felids. Zoo Biology, 22: 189-198. 
Dirección General de Zoológicos de la Ciudad de México. 2006. Los zoológicos de la ciudad de México ayer y hoy. Edición digital a texto completo. In: www.sma.df.gob.mx/sma/download/archivos/zoologicos/06.pdf

Dirección General de Zoológicos de la Ciudad de México. 2007. Primer Informe de Trabajo de la Secretaría del Medio Ambiente 2007. Edición digital a texto completo. In: http://www.sma.df.gob. mx/sma//links/download/archivos/1er_informe_sma_2007/05zoologicos.pdf

Granda-Serrano, C. E. \& Z. E. Romo-Graniel. 2004. Enriquecimiento ambiental para jaguares (Panthera onca) en cautiverio en el Parque Museo de la Venta, Villahermosa Tabasco. Tesis de Licenciatura, Universidad Autónoma de Tabasco. Villahermosa, Tabasco. 45 pp.

Jones, M. K., Marno, R. \& H. Buchanan-Smith. 2005. Novel feeding and hunting enrichment for large captive felids: the lionrover and responsive hanging prey. Proceedings of the Seventh Annual Symposium on Zoo Research: 91-101.

Mallapur, A. 1999. Environmental influences on space utilization and the activity budget of captive leopards (Panthera pardus fusca) in five zoos in Southern India. Tesis de Maestría, Saurashtra University, India.

Mallapur, A. \& R. Chellam. 2002. Environmental influences on stereotypy and the activity budget of Indian leopards (Panthera pardus) in four zoos in Southern India. Zoo Biology, 21: 585-595.

Martin, P. \& P. Bateson. 1997. Measuring behaviour. An introductory guide. Cambridge University Press. Londres.

Martínez, G. 2006. ¿Elefantes Pintando? Una efectiva terapia ocupacional. Revista Electrónica de Veterinaria REDVET. 06. España. Edición digital a texto completo In: http://www.veterinaria.org/revistas/redvet/n090906.html

Margulis, S. W., Hoyos, C. \& M. Anderson. 2003. Effect of felid activity on zoo visitor interest. Zoo Biology, 22: 587-599.

Mason, G. J. 1991. Stereotypies: a critical review. Animal Behaviour, 41: 1015-1037.

Mason, G., Clubb, R., Latham, N. \& S. Vickery. 2007. Why and how should we use environmental enrichment to tackle stereotypic behaviour? Applied Animal Behaviour Science, 1002: 163-188.

McPhee, E. 2002. Intact carcasses as enrichment for large felids: Effects on on- and off-exhibit behaviors. Zoo Biology, 21: 37-47.

Mellen, J., Hayes, M. \& D. Shepherdson. 1998. Captive environments for small felids, pp. 184-201. In: D. Shepherdson, J. Mellen \& M. Hutchins (Eds.). Second nature: environmental enrichment for captive animals. Washington: Smithsonian Institution Press.

Mellen, J. \& M.S. McPhee. 2001. Philosophy of environmental enrichment: past, present, and future. Zoo Biology, 20: 211-226.

Shepherdson, D. 1998. Tracing the path of environmental enrichment in zoos, pp. 1-12. In: D. Shepherdson, J. Mellen \& M. Hutchins (Eds.). Second nature: environmental enrichment for captive animals. Washington: Smithsonian Institution Press.

Shyne, A. 2006. Meta-analytic review of the effects of enrichment on stereotypic behavior in zoo mammals. Zoo Biology, 25: 317-337.

Skibiel, A., Trevino, H. \& K. Naugherz. 2007. Comparison of several types of enrichment for captive felids. Zoo Biology, 26: 371-381.

Swaisgood, R. R. \& D. J. Shepherdson. 2005. Scientific approaches to enrichment and stereotypies in zoo animals: what's been done and where should we go next? Zoo Biology, 24: 499-518.

Tarou, L. R., Bloomsmith, M. A. \& T. L. Maple. 2005. Survey of stereotypic behaviours in prosimians. American Journal of Primatology, 65: 181-196.

WAZA (World Association of Zoos and Aquariums). 2005. The World Zoo Conservation Strategy: Building a Future for Wildlife. World Aquaria and Zoos Association Executive Office, Bern.

Zar, J. 1999. Biostatistical Analysis. Fourth edition. Prentice Hall. New Jersey. U.S.A. 